\title{
THE OCTOBER MEETING OF THE AMERICAN MATHEMATICAL SOCIETY
}

The two hundred nineteenth regular meeting of the Society was held at Columbia University on Saturday, October 29, 1921, extending through the usual morning and afternoon sessions. The attendance included the following forty members:

Archibald, Babb, Blake, Bowden, Cole, G. M. Conwell, Eisenhart, Fite, Philip Franklin, Gill, Glenn, Gronwall, Grove, Haskins, Hebbert, Joffe, Kasner, Kellogg, Kircher, Kline, Lamson, McDonnell, MacDuffee, MacNeish, H. H. Mitchell, Northcott, Oglesby, Pfeiffer, Post, Reddick, R. G. D. Richardson, Ritt, Seely, Siceloff, P. F. Smith, Veblen, H. S. White, Whittemore, E. C. Williams, J. W. Young.

At the meeting of the Council, the following thirty persons were elected to membership in the Society:

Mr. Clarence Raymond Adams, Harvard University;

Professor Edward Tankard Browne, Trinity College;

Professor Frank William Bubb, Washington University;

Mr. Curtis Corsair Carter, Bluffs, Scott County, Ill.;

Mr. Joel David Eshleman, University of Pennsylvania;

Mr. Matthew Moses Feldstein-Tartakovsky, Quartermaster Corps, U. S. Army;

Mr. Harold Joseph Gay, Worcester Polytechnic Institute;

Professor Lachlan Gilchrist, University of Toronto;

Professor Edward Sanford Hammond, Bowdoin College;

Mr. Edward H. Hezlett, Connecticut General Life Insurance Company;

Professor Joseph Ellis Hodgson, West Virginia University;

Miss Nelle Louise Ingels, Bureau of Statistics, Interstate Commerce Commission;

Mr. John Melvin Laird, Connecticut General Life Insurance Company;

Mr. Rudolph Ernest Langer, Harvard University;

Professor Michael Alexander Mackenzie, University of Toronto;

Professor Edmund Sewall Manson, Ohio State University;

Mr. Louis Edward Mensenkamp, High School, Freeport, Ill.;

Dr. Raymond Kurtz Morley, Worcester Polytechnic Institute;

Miss Eleanor Pairman, Broomieknowe, Scotland;

Miss Echo Dolores Pepper, University of Washington;

Mr. Charles Scott Porter, Worcester Polytechnic Institute;

Professor William Walter Rankin, Agnes Scott College; 
Mr. Noah Bryan Rosenberger, Philadelphia, Pa.;

Mr. Norris Edward Sheppard, University of Toronto;

Professor George Eulas Foster Sherwood, University of California, Southern Branch;

Miss May Julia Sperry, Knox College;

Professor John Lighton Synge, University of Toronto;

Professor John Sidney Turner, Alabama Polytechnic Institute;

Mr. Correa Moylan Walsh, Bellport, L. I.;

Professor Thomas Orr Walton, Kalamazoo College.

One application for membership was received.

A committee consisting of Mr. S. A. Joffe and Professor P. H. Linehan was appointed to audit the accounts of the Treasurer for the current year. A list of nominations of officers and other members of the Council was adopted and ordered printed on the official ballot for the annual election. It was voted to publish a List of Officers and Members for 1921-1922.

It was voted to accept the invitation of Harvard University to hold the annual meeting of 1922 at Cambridge.

Vice-president Cole presided at the morning session of the Society, relieved by Professor P. F. Smith in the afternoon. Titles and abstracts of the papers read at this meeting follow below. The papers of Mr. Rice, Dr. Walsh, Mr. Robinson, and Dr. Campbell were read by title. Professor R. L. Moore's papers were read by Professor Kline.

1. Professor J. K. Whittemore: Total geodesic curvature.

The author shows that the rate of turning, per unit length of arc of a curve on a surface, of the plane determined by the tangent to the curve and the normal to the surface (called by Professor Osgood* the bending of the curve) is given by

$$
\sqrt{1 / \rho_{g}{ }^{2}+1 / \tau_{\theta}^{2}}
$$

where $\rho_{g}$ and $\tau_{g}$ are the radii of geodesic curvature and geodesic torsion respectively.

2. Professor J. F. Ritt: On the composition of polynomials.

A polynomial $F(z)$ of degree greater than unity is here called composite if there exist two polynomials $\varphi_{1}(z)$ and $\varphi_{2}(z)$, each of degree greater than unity, such that $F(z)=\varphi_{1}\left[\varphi_{2}(z)\right]$.

\footnotetext{
* This Bulletin, vol. 27, p. 391.
} 
Otherwise $F(z)$ is called prime. Consider a decomposition of $F(z)$ into prime polynomials,

$$
F=\varphi_{1} \varphi_{2} \cdots \varphi_{r}
$$

in which each $\varphi_{i}(z)$ is understood to be substituted for $z$ in the polynomial which precedes it. Two such decompositions are considered equivalent if the second can be obtained from the first by inserting suitable linear polynomials between the prime polynomials. In this paper the circumstances are completely determined under which a polynomial can have two distinct decompositions into prime polynomials. If there are two such distinct decompositions, they contain the same number of polynomials, and the degrees of the polynomials in one decomposition are the same as those in the other, except for the order in which they occur. The full statement of results is simple, but somewhat too long for an abstract.

3. Professor J. F. Ritt: Complete determination of polynomials whose inverses can be expressed in terms of radicals.

In an earlier paper the writer determined the structure of those Riemann surfaces with a prime number of sheets which support functions expressible in terms of radicals. For equations of composite degree, this paper determines all polynomials whose inverses can be expressed in terms of radicals. If a polynomial $F(z)$, with inverse so expressible, is decomposed into prime polynomials, in the manner explained in the preceding abstract, then each prime polynomial is either of the fourth degree, or else is of the form $\lambda_{1}\left\{\pi\left[\lambda_{2}(z)\right]\right\}$, where $\lambda_{1}(z)$ and $\lambda_{2}(z)$ are linear, and where $\pi(z)$ is either a prime power of $z$, or else a trigonometric polynomial of prime degree.*

4. Professor R. L. Moore: Concerning continuous curves in the plane.

In this paper the following theorems are established: (I) If $N$ is a closed proper subset of a continuous curve $M$ and two points of $M-N$ can be joined by a connected subset of $M-N$ then they can be joined by a simple continuous arc which is a subset of $M-N$; (II) If, in a plane, two points are separated from each other by a continuous curve $M$ then they are separated from each other by a simple closed curve which is a subset of $M$. The first of these theorems is an extension of a theorem proved in a former paper (this Bulletrin, vol. 23 (1917), pp. 233-236).

\footnotetext{
${ }^{*} \pi(z)$ is a trigonometric polynomial of degree $n$ if $\cos n u=\pi(\cos u)$.
} 
5. Professor R. L. Moore: Concerning the relation of a continuous curve to its complement in space of three dimensions.

According to a theorem of Schoenflies, in order that a point set $M$ lying in a plane $S$ should be a continuous curve it is necessary and sufficient that (1) every point of the boundary of any one of the domains complementary to $M$ should be reachable "from all sides" with respect to that domain, (2) if $\epsilon>0$ there do not exist in $S-M$ infinitely many mutually exclusive domains $R_{1}, R_{2}, R_{3}, \cdots$ all of diameter more than $\epsilon$ and such that each $R_{n}$ has a portion of $M$ as its boundary. In the present paper an example is given of a continuous curve in space of three dimensions which satisfies neither of these conditions. It is also shown that a similar criterion of Jordan and Schoenflies that a plane point set of more special type be a continuous curve is in the case of three dimensions neither necessary nor sufficient. It is true, however, that a closed, bounded and connected point set in a space of three dimensions is a continuous curve provided it is the common boundary of two mutually exclusive domains both of which are uniformly connected im kleinen. It is not sufficient to assume merely that one of these domains has this property.

6. Professor Edward Kasner: An algebraic solution of Einstein's cosmological equations.

Professor Kasner discusses the gravitational equations in Einstein's latest cosmological form $G_{i k}-\frac{1}{4} g_{i k} G=0$. If we require the quaternary form $d s^{2}$ to be the sum of two binary forms, that is, the sum of the squared elements of two surfaces, then the only cosmological solution (neglecting the trivial euclidean form) is found to be

$$
d s^{2}=x_{1}^{-2}\left(d x_{1}^{2}+d x_{2}^{2}\right)+x_{3}^{-2}\left(d x_{3}^{2}+d x_{4}^{2}\right) .
$$

This represents a quartic manifold of four dimensions embedded in a 6-flat. The finite equations, in six cartesian coördinates, are

$$
X_{1}^{2}+X_{2}^{2}+X_{3}^{2}=1, \quad X_{4}^{2}+X_{5}^{2}+X_{6}^{2}=1 .
$$

This is probably the simplest solution of Einstein's equations which has thus far been found, and the first one (beyond the obvious flat and spherical spaces) which in its finite form is algebraic. In an earlier paper solutions have been found where the ten $g_{i k}$ are algebraic functions, but these do not usually represent algebraic spreads. (See ScIEnce, Sept. 30, 1921, pp. 304-305, and a forthcoming paper in the MATHEmatische AnNalen.) 
7. Dr. T. H. Gronwall: On biharmonic functions.

A biharmonic function satisfies the equation $\Delta \Delta u=0$ or $\partial^{4} u / \partial x^{4}+2 \partial^{4} u / \partial x^{2} \partial y^{2}+\partial^{4} u / \partial y^{4}=0$ and is regular inside a curve $C$, the values of $u$ and $\partial u / \partial n$ on $C$ being given. Dr. Gronwall proves that when $\partial u / \partial n \geqq 0$ everywhere on $C$, then $u$ has at least one maximum but no minimum inside $C$. A number of other qualitative results are also proved, most of which have immediate physical applications. Some of these results were conjectured, but not proved, by Hadamard in his prize memoir on elastic plates (MÉmoIRES DES SAVANTs ÉTRANGERS, 1909).

8. Mr. L. H. Rice: General formulation of a combinatory method used by William Emerson and others.*

The method consists in so interpreting a given problem in combinations, in terms of element, entity, subclass, and subdivision, that the problem shall take the following form: Given, a stock containing $k$ subclasses each included in the next, the $i$ th subclass being composed of $\alpha_{i}$ elements. Required, the number ' $C$ of combinations, each separable into $k$ mutually exclusive subdivisions, the $i$ th subdivision being composed of $\beta_{i}$ elements taken from among the $\alpha_{i}$ elements (or composed of $\beta_{i}$ entities which are in one to one correspondence with $\beta_{i}$ of the $\alpha_{i}$ elements of the stock). The answer to the problem is then apparent:

$C=\left(\begin{array}{c}\alpha_{1} \\ \beta_{1}\end{array}\right)\left(\begin{array}{c}\alpha_{2}-\beta_{1} \\ \beta_{2}\end{array}\right)\left(\begin{array}{c}\alpha_{3}-\beta_{2}-\beta_{1} \\ \beta_{3}\end{array}\right) \cdots\left(\begin{array}{c}\alpha_{k}-\beta_{k-1}-\cdots-\beta_{1} \\ \beta_{k}\end{array}\right)$.

9. Dr. J. L. Walsh: A theorem on loci connected with crossratios.

The following theorem, formerly proved for real $\lambda$, is now extended to non-real $\lambda$ : If the respective loci of the points $z_{1}, z_{2}, z_{3}$ are circular regions, then the locus of the point $z_{4}$ determined by the constant cross-ratio $\lambda=\left(z_{1}, z_{2}, z_{3}, z_{4}\right)$ is also a circular region.

* (1) William Emerson, The Doctrine of Combinations, Permutations, and Composition of Quantities, London, 1770. (2) L. Oettinger, ARCHIV FǗr Mathematik, 15 (1850), p. 241, § 6. (3) L. H. Rice, Coefficient of the general term in the expansion of a product of polynomials, this BuLLETIN, vol. 27 (1921), p. 344. 
10. Mr. L. B. Robinson: A generalization of the notion of covariants.

Riquier has generalized the theory of complete systems. Mr. Robinson shows that a generalized complete system

$$
\frac{\partial w_{i}}{\partial x_{j}}=\sum_{r=1}^{n} A_{r j} \frac{\partial w_{i}}{\partial t_{r}}+U_{i j}
$$

can be utilized to generalize the notion of covariants. The generalized covariants are solutions of the above system and can be obtained by a finite number of differentiations or integrations.

11. Dr. G. A. Campbell: Inductances of grounded circuits.

Any network of conductors located on the surface of the earth with which it is conductively connected at any number of points will, for direct currents, have self and mutual inductances which are equal to the Neumann integral extended over the network alone. In other words, that portion of the complete Neumann integral for closed circuits which involves the return currents through the earth vanishes. The earth is assumed to be flat, of infinite extent, of unit permeability and of uniform conductivity.

R. G. D. Richardson, Secretary.

\section{THE OCTOBER MEETING OF THE SAN FRANCISCO SECTION}

The thirty-eighth regular meeting of the San Francisco Section was held at the University of California on Saturday, October 22, 1921. Professor Lehmer presided at the earlier part of the meeting, later relieved by Professor Allardice. The total attendance was twenty-five, including the following seventeen members of the Society:

Alderton, Allardice, Bernstein, Blichfeldt, Buck, Cajori, Daus, Edwards, Haskell, Hoskins, Lehmer, Moreno, F. R. Morris, Noble, T. M. Putnam, Pauline Sperry, A. R. Williams.

The following officers were elected for the year: Chairman, Professor Allardice; Secretary, Professor Bernstein; programme committee, Professors Blichfeldt, Lehmer, Bernstein. 